Proceedings of the 2010 Winter Simulation Conference

B. Johansson, S. Jain, J. Montoya-Torres, J. Hugan, and E. Yücesan, eds.

\title{
TO AGENT-BASED SIMULATION FROM SYSTEM DYNAMICS
}

\author{
Charles M. Macal \\ Argonne National Laboratory \\ Center for Complex Adaptive Agent Systems Simulation $\left(\mathrm{CAS}^{2}\right)$ \\ 9700 S. Cass Ave. \\ Argonne, IL 60439, USA
}

\begin{abstract}
Agent-based simulation (ABS) is a recent modeling technique that is being widely used in modeling complex social systems. Forrester's System Dynamics (SD) is another longstanding technique for modeling social systems. Several classical models of systems, such as the Kermack-McKendrick model of epidemiology, the Lotka-Volterra equations for modeling predator-prey relationships, and the Bass model for innovation diffusion are formulated as systems of differential equations and have corresponding System Dynamics representations as difference equations. The ABS and SD modeling approaches take fundamentally different perspectives when modeling a system, which can be characterized as bottom-up (ABS) versus top-down (SD). Yet many systems can be equivalently modeled by either approach. In this paper, we present a formal specification for SD and ABS models, use the specification to derive equivalent ABS representations, and present an example of an SIR epidemic model having SD and ABS counterparts.
\end{abstract}

\section{INTRODUCTION}

Forrester's System Dynamics (SD) is a commonly used systems modeling technique with a long tradition of applications to social systems (Forrester 1961; Forrester 1971; Roberts et al. 1983, Sterman, 2000). Systems Dynamics models consist of a set of difference equations (similar to differential equations but with a fixed time step) that are recursively solved forward through time. In general, SD models consist of a relatively small number of state variables that completely define the state of the system being modeled, and a specification of the rate of change of each of the state variables, which depends on the previous system state. SD has its roots in dynamic systems and control theory, and emphasizes the importance of feedback effects between aggregate level system components as strong determinants of system behavior.

Agent-based simulation (ABS) is a relatively recent modeling technique that is being widely used to model complex systems composed of interacting, autonomous "agents" (Epstein and Axtell 1996, Bonabeau 2001, North and Macal 2007, Macal and North 2009). Agents have behaviors, which are often described by simple rules. Agents interact with and influence each other, learn from their experiences, and adapt their behaviors so they are better suited to their environment. By modeling agents individually, the full effects of the diversity that exists among agents with respect to their attributes and behaviors can be observed as they give rise to the dynamic behavior of the system as a whole.

The ABS and SD modeling approaches take fundamentally different perspectives when modeling a system, which has been characterized as bottom-up (ABS), i.e., modeling a system by modeling the individual entities that compose the system and their interactions, versus top-down (SD), i.e., modeling a system by breaking it into its major components and modeling the component interactions. Yet many systems can be modeled by either the SD or ABS approach, as has been noted by several researchers. For example, Scholl (2001) describes agent-based modeling and System Dynamics as two prominent techniques for modeling nonlinear systems and identifies areas in which the two modeling traditions overlap as well as complement each other, concluding with a call for more research on the subject. Comparing the 


\section{Macal}

SD and ABS approaches to modeling the same problem has been addressed by Parunack (1998) in modeling supply chains, Marin et al. (2006) in work force modeling, and Norlin (2007) in modeling ecologies, among others.

Several classical system models, such as the Kermack-McKendrick model of epidemic dynamics (Kermack and McKendrick 1927), the Lotka-Volterra equations for modeling predator-prey relationships, the Bass model for innovation diffusion, chemical kinetics models, and many others are formulated as systems of ordinary differential equations and have corresponding System Dynamics representations as difference equations. It is well-known that the Kermack-McKendrick model in particular can be formulated as an agent-based simulation also, and produces similar results to an SD formulation (Bagni et al. 2002, Borshchev and Filippov 2004, Wakeland et al. 2004, Epstein et al. 2008, Rahmandad and Sterman 2008). In this paper, we elaborate on this similarity and describe the specifics of how to transform a System Dynamics model into an "equivalent" ABS model. By equivalent, we mean that both models represent the same system down to its detailed specifications using the fullest capabilities that each approach can provide. The equivalent SD and AB models are different in form and do not necessarily produce the same numerical results given identical assumptions. We will illustrate and elaborate on this equivalency by describing how to transform the Kermack-McKendrick SIR model of epidemic dynamics into an agent-based simulation of the same system. The transformation process is a general one, however, and should be applicable to any SD model that has strong component, or agent, interactions.

This paper is organized as follows. In Section 2 we provide useful definitions of SD models and the subset of the type of agent-based models considered in this paper. Section 3 describes the procedure for transforming an SD model into two equivalent ABS representations. Section 4 presents comparative model results, and we conclude with directions for further work in the area.

\section{DEFINITIONS OF SD AND ABS MODELS}

In this section we demonstrate the equivalence between a certain kind of agent-based model and a System Dynamics version of the model. First we establish a formalism for the two approaches.

\subsection{Definition of System Dynamics Model}

We formally define a System Dynamics model, SDM, as a triple:

$$
\mathrm{SDM}=\left\{\mathrm{S}_{\mathrm{t}}, \mathrm{R}_{\mathrm{t}}\left(\mathrm{S}_{\mathrm{t}-1}\right), \mathrm{T}\right\}
$$

where: $S_{t}$ is the set of state variables at time $t, R_{t}(S)$ is the set of rate variables at time $t$, dependent on the state variables from the previous one (or possibly more) time periods, and $\mathrm{T}$ is the "temporal" simulation engine, the mechanism that steps the model through time. $\mathrm{T}$ is logically simplistic in that it simply implements updating the simulation time (the simulation clock) from time $t$ to $(t+\Delta t)$ and updating the values of the state and rate variables. The standard SD specification also includes "auxiliary" equations and variables defined for convenience by partitioning the space of rate variables. For purposes of this discussion, we assume the state equations are in reduced form, devoid of auxiliary variables and auxiliary equations. Implicitly throughout, we also assume that the SD models of interest here are ones that include some elements of decision making behavior, as opposed to models of purely physical processes, such as models of world weather systems or climate dynamics. Such SD models allow comparisons to be made with agentbased models.

Solving a System Dynamics model is equivalent to solving a set of ordinary differential equations using the forward Euler method in which the step size, $\Delta \mathrm{t}$, is set to one (Ascher and Petzold 1998). In general, the solution of a set of difference equations, such as comprising a System Dynamics model, is not equivalent to a solution obtained for the corresponding differential equation model, but there special cases exist in which the equivalence does hold (Mickens 2002, Letellier et al. 2004). We make these comparisons for the SIR epidemic model addressed later in the paper. 


\section{Macal}

\subsection{Definition of Agent}

Although there is not universal agreement on the definition of an agent, in part because it depends on the use of the model, from a practical modeling standpoint, agents have certain essential characteristics to qualify as agents (Macal and North 2010):

- An agent is a self-contained, modular, and uniquely identifiable individual. The modularity requirement implies that an agent has a boundary and one can easily determine whether something is part of an agent, is not part of an agent, or is a shared attribute.

- An agent is autonomous and self-directed. An agent can function independently in its environment and in its interactions with other agents, at least over a limited range of situations that are of interest in the model. An agent has behaviors that relate information sensed by the agent to its decisions and actions.

- An agent has a state that varies over time. Just as a system has a state consisting of the collection of its state variables, an agent also has a state that represents the essential variables associated with its current situation. An agent's state consists of a set or subset of its attributes. The state of an agent-based model is the collective states of all the agents along with the state of the environment. An agent's behaviors are conditioned on its state.

- An agent is social, having dynamic interactions with other agents that influence its behavior.

Agents may also have other optional but useful characteristics, such as being adaptive, goal-directed, and heterogeneous across the population of agents. For the purposes of the models discussed in this paper, in addition to the essential characteristics noted above, only the heterogeneity aspect of agents is needed.

\subsection{Definition of Agent-based Model}

We define an Agent-based Simulation model, ABSM, as a triple:

$$
\operatorname{ABSM}=\left\{A_{t}, M\left(A_{t-1}\right)_{t}, I\right\},
$$

where: $A_{t}$ is the set of agents including their states at time $t, M\left(A_{t-1}\right)$ is the set of mechanisms that operate on the agents in groups or individually at time $t$, which is specified as dependent on the states of the agents from the previous time period(s), and I is the agent interaction protocol. An example of I would be the procedure for determining which agents act and interact at any point in time. It should be noted that this particular specification ABSM is not meant to be a completely general specification for all ABS; here, we only consider the subclass of ABS in which agent actions and interactions occur at fixed time intervals (i.e., time-stepped simulation) for purposes of more direct comparison with the SD approach. A more general agent interaction protocol would be one in which agents actions and interactions occur in response to certain endogenous conditions in the simulation. There are many agent-based simulation in which these complexities are essential parts of the agent model.

We next state a formal result on the relationship between SD and ABS models reproduced from Macal (2006).

\subsection{Equivalence Theorem}

Agency Theorem for Systems Dynamics: Let $S$ be the set of all System Dynamics models $S D M=\left\{S_{t}\right.$, $\left.\mathrm{R}_{\mathrm{t}}\left(\mathrm{S}_{\mathrm{t}-1}\right), \mathrm{T}\right\}$ and let $\mathrm{S}_{\mathrm{t}+1}=\mathrm{ESDM}\left[\mathrm{S}_{\mathrm{t}}\right]$ be the equivalent iterated map representation for SDM. Assume ESDM is invertible. Let $A$ be the set of all Agent-based Simulation models $A B S=\left\{A_{t}, M_{t}\left(A_{t-1}\right), I\right\}$. Then, $\mathrm{S}$ is a strict subset of $\mathrm{A}$ such that $\mathrm{S} \square \mathrm{A}$.

Proof: First, we note that assuming invertibility of ESDM, any system of state and rate equations can be resolved explicitly for the subset of variables that are agent decision variables as functions of the remain- 


\section{Macal}

ing system state variables. If agent decision variables are not explicitly defined, they may be introduced into the model through the use of auxiliary variables. To prove the theorem it suffices to note that the set of agent interaction mechanisms that define agent interactions at specific discrete times, I, includes the time advancement mechanism $\mathrm{T}$.

The theorem simply states that every well formulated SD model has an equivalent formulation as an ABS model of the class of time-stepped ABS models considered here. In the following section, we derive two corresponding agent-based models from an SD formulation of the Kermack-McKendrick SIR model.

\section{TRANSFORMING AN SD MODEL INTO AN EQUIVALENT ABS}

\subsection{The SIR Model}

The SIR model was originally proposed by Kermack and McKendrick (1927) and has been used widely to understand and predict the spread of actual epidemics. The population is divided into three groups or compartments consisting of susceptible individuals, denoted by S, infected individuals, denoted by I, and recovered individuals, denoted by $\mathrm{R}$ (thus $\mathrm{S}-\mathrm{I}-\mathrm{R}$ ):

$$
\begin{aligned}
& \frac{d S}{d t}=-\beta S I / N \\
& \frac{d I}{d t}=\beta S I / N-\gamma I \\
& \frac{d R}{d t}=\gamma I
\end{aligned}
$$

Initial conditions: $S_{0}=N-1, I_{0}=1, R_{0}=0$.

where $\beta$ is related to the number of contacts an individual has with other individuals and the likelihood that an infected individuals transmits the infection to a susceptible individual upon contact, $\gamma$ is the rate at which infected individuals recover from an infection, which is taken as $1 /$ (mean duration of illness), and $\mathrm{N}$ is the population size, assumed to be constant in this basic representation. In the standard SIR model, the initial conditions for the population consist of one infected individual and no recovereds.

The SIR model is also referred to as the homogeneous mixing model because of three implicit assumptions in the formulation: 1) the population is fully mixed, meaning that individuals with whom a susceptible individual has contacts are chosen at random from the whole population, 2) all individuals have approximately the same number of contacts in the same period of time, and 3) all contacts transmit the disease with the same probability. All infected individuals are assumed to transmit the disease to the same number of people, and the all susceptible people have the same chance of becoming infected. A number called the basic reproduction number $\mathrm{R} 0$, which is the initial value of $\mathrm{d} / \mathrm{dt}$, is often used to indicate the initial severity of an epidemic.

Equation system (1) represents the number of susceptibles that become infected in the time interval $\Delta \mathrm{t}$. Let $\Delta \mathrm{S}$ be the number of susceptibles becoming infected in $\Delta \mathrm{t}$. Then, $\Delta \mathrm{S}=$ (number of susceptibles, $\mathrm{S}$ ) $\times \operatorname{Pr}[$ Susceptible becomes infected in $\Delta \mathrm{t}$ ], where $\operatorname{Pr}[$ Susceptible becomes infected] $=\operatorname{Pr}[$ Susceptible contacts an infected] $\times \operatorname{Pr}[$ infection is transmitted from an Infected to a Susceptible upon contact], where $\operatorname{Pr}[$ Susceptible contacts an infected $]=($ Number of contacts per individual $) \times \operatorname{Pr}[$ A contacted individual is infected], where $\operatorname{Pr}$ [A contacted individual is infected] = I / (Number of individuals in the population, $\mathrm{N}$ ).

Therefore, $\Delta S=($ Number of susceptibles, $S) \times($ Number of contacts per individual $) \times I /($ Number of individuals in population, $\mathrm{N}) \times \operatorname{Pr}$ [infection is transmitted from an infected individual to a susceptible individual upon contact] $\Delta \mathrm{S}=\mathrm{S} \times$ (number of contacts per individual) $\times \mathrm{I} / \mathrm{N} \times \operatorname{Pr}$ [infection is transmitted from an infected individual to a susceptible individual upon contact] $\Delta \mathrm{S}=$ (Number of contacts per indi- 


\section{Macal}

vidual $) \times \operatorname{Pr}$ [infection is transmitted from an infected individual to a susceptible individual upon contact] $\times \mathrm{S} \mathrm{I} / \mathrm{N}$.

Hence, as noted by Sterman (2000), $\beta$ in (1) is a composite of two factors, the number of contacts per individual, $\beta_{c}$, and the probability that the infection is transmitted from an infected individual to a susceptible individual upon contact, $\beta_{I}$, as in:

$$
\beta=\beta_{c} \beta_{i}
$$

Whereas, in (2) the composite of $\beta_{c}$ and $\beta_{i}$ appears in the standard SIR model (1), $\beta_{c}$ and $\beta_{i}$ are treated separately in the agent-based SIR model, Model 2, as described below.

Note, in this derivation the number of contacts per individual is assumed to be a constant for all disease states. That is, an infected individual has as many contacts with others as does a susceptible individual. For a constant population size, $\mathrm{N}=\mathrm{S}+\mathrm{I}+\mathrm{R}$. The output of a typical solution of the SIR model in (1) is shown in Figure 1. The three population states (numbers of susceptible, infected, and recovered individuals) are shown as they vary over time. The output shows an epidemic, as the entire population of agents becomes infected and the number of susceptible individuals declines to zero over the course of the simulation. Note, the smooth nature of the curves due to the deterministic nature of the model and the meanfield characterizations of agent interactions. Key statistics that one might be interested in from such a simulation are the peak number of infected individuals and the time at which the peak occurs. For this simulation run with a population size of 1000 , the peak number of infected individuals is 593 occurs at time 21.

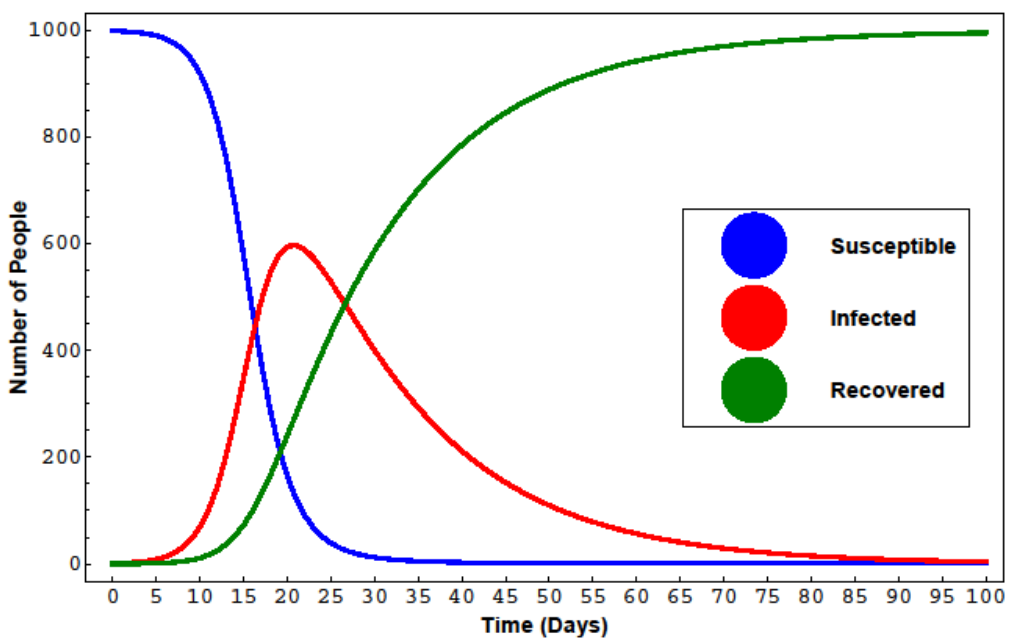

Figure 1: Typical SIR model solution showing progression of population disease states for susceptible, infected, and recovered compartments. In this example, the entire population becomes infected and eventually recovers.

\subsection{The System Dynamics SIR Model}

The System Dynamics version of the SIR model is derived by substituting into (1):

$$
\frac{d S}{d t}=\frac{\Delta S_{t+1}-\Delta S_{t}}{\Delta t}
$$

which results in a system of difference equations: 


$$
\begin{gathered}
\text { Macal } \\
S_{t+1}=S_{t}-\left(\beta S_{t} I_{t} / N\right) \Delta t \\
I_{t+1}=I_{t}+\left(\beta S_{t} I_{t} / N-\gamma I_{t}\right) \Delta t \\
R_{t+1}=R_{t}+\left(\gamma I_{t}\right) \Delta t \\
\text { Initial conditions: } S_{0}=N-1, I_{0}=1, R_{0}=0 .
\end{gathered}
$$

System (3) has a solution for $\Delta \mathrm{t}=1$, which is a common setting for a System Dynamics model, that is reasonably close to the solution of the original differential equation system (1); and as $\Delta t \rightarrow 0$, the solution of (3) approaches the solution of (1). For small $\Delta t$, solving (3) recursively for $t=0,1,2, \ldots$ is equivalent to applying the forward-Euler method to (1), a common method of solving ordinary differential equations numerically.

\subsection{Agent-based SIR Model 1}

We will consider two formulations of the agent-based SIR model, the compartmentalized ABS model, Model 1, and the individual-based ABS SIR Model, Model 2. Model 1 is something of a "naïve" agentbased model. Although the agents in Model 1 satisfy the criteria for it to be an agent-based model as stated in Section 2.2 and provides equivalent results to the SD model (results from all models are compared below), it provides no additional information or implementation advantages over the SD model. It is included here for the sake of illustration. (Model 2 is a fully individual-based agent model and provides additional information over the SD model.)

In the following description, we specify the models in pseudo-code, taking a decidedly objectoriented programming approach, in which agent types are defined as classes. Agent interactions are specified by methods defined at the class level. Methods operate on the agent instances in the corresponding agent classes to model agent interactions (in the case of the SIR model agent interactions are contacts) and to update the agent states through time.

For Model 1, we define two agent classes in pseudo-code consisting of the population, with population parameters, and agent compartments consisting of all individuals in a particular disease state:

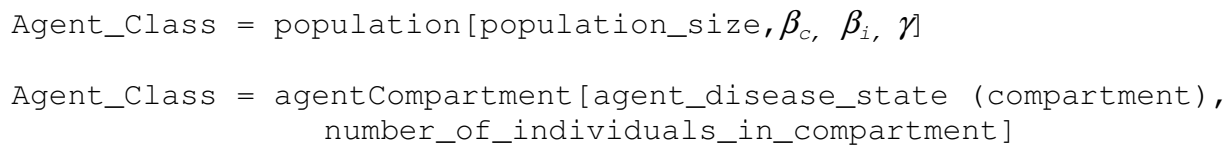

For agent compartments, the state of an agent at any time is the number of agents in the particular disease state. The agent compartment state is the only information in the model that is dynamically updated. All other parameters are static.

Based on the agent class definitions above, there is one population instance, with total population $\mathrm{N}$, and three agent compartment instances, corresponding to disease states S, I, and R:

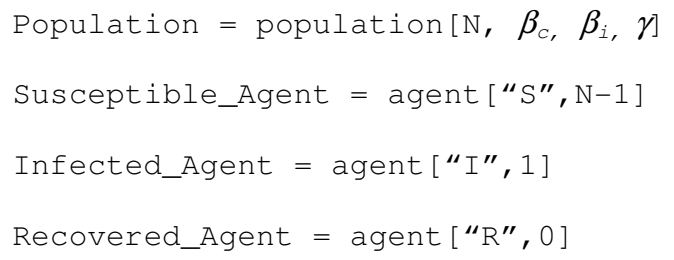

We define the following class-specific methods that operate on the instances, one method per term in the disease state transition equations, to complete the model specification. The following pseudo-code defines these methods.

Method_Susceptible_To_Infected (remove $\beta$ IS/N individuals from $S$ ) =( 


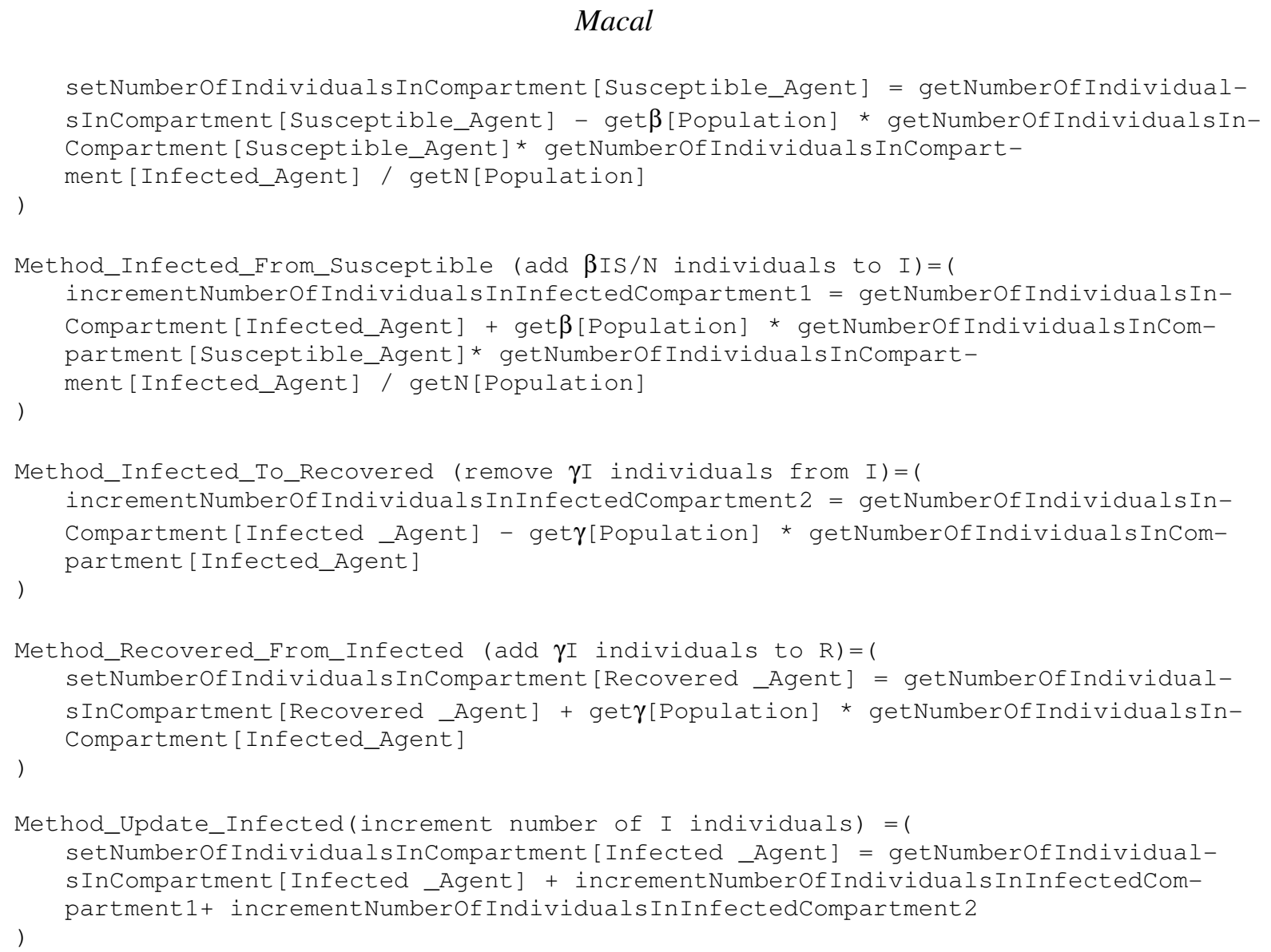

In the agent-based formulation Model 1, the methods essentially define agent compartment behaviors, but the behaviors are rather vacuous. For example, the behavior for the susceptible compartment agent, Method_Susceptible_To_Infected, simply reduces the number of susceptible agents in the susceptible agent compartment. Implicit in this formulation is the connectivity between compartments. That is, $\mathrm{S}$ sends individuals to I, I receives individuals from $\mathrm{S}$, I sends individuals to $\mathrm{R}$, and $\mathrm{R}$ receives individuals from R.

To complete the description of Model 1, we would set up a time-stepped simulation in which the methods operate on the agent instances at each time step and all the agent compartment states are updated.

We assert without proof that the ABS Model 1 produces exactly the same results for S, I, and R over time as does the SD model for a fixed-time step, $\Delta \mathrm{t}$, of length one.

In addition, we note that ABS Model 1 is easily extensible to finer and finer levels of granularity with respect to the compartments specified. For example, one may want to include activity states (home, school, work, travel, etc.) as well as disease states for the agents and specify compartment-specific contact and transmission rates. One would then define activity-disease state compartments accordingly. Adding compartments to the model would correspond to adding equations to the SD formulation.

If the transitions of agents between compartments are specified probabilistically, however, ABS Model 1 is readily extended to a be stochastic model. If compartment (state) transitions are probabilistic and not conditioned on past states, ABS Model 1 becomes a sort of Markov chain model. This is a modeling approach similar to that of Larsen (2007) for modeling heterogenous populations, and adopted by Aleman, Wibisono, and Schwartz (2009) in their recent agent-based model of pandemic influenza.

\subsection{Agent-based SIR Model 2}

For Model 2, we define a single agent class in pseudo-code that covers any individual in the population: 


\section{Macal}

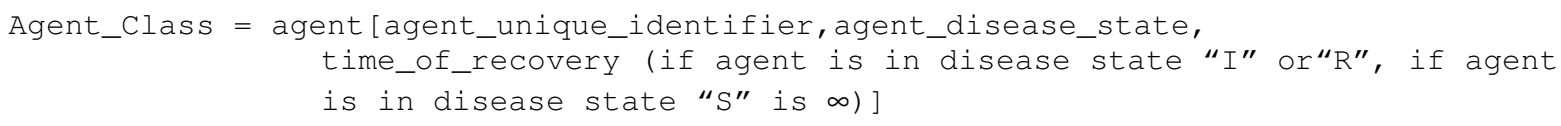

The state of an agent at any time is its particular disease state. The agent state is the only information in the model that is dynamically updated. All other parameters are static. Comparing with Model 1, in Model 2, the number of individuals in the population (in each disease state) is implied by the number of agents (in each disease state).

Based on the agent class definitions above, there are $\mathrm{N}$ agent instances in various disease states of $\mathrm{S}$, $\mathrm{I}$, and $\mathrm{R}$, where $\mathrm{N}$ is the total population size. For example, denoting a list of items as enclosed in braces, an example of agent instances at initial time $\mathrm{t}_{0}$ is:

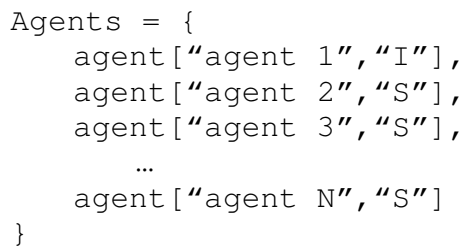

In Model 2, the process for updating the agent states is more complex than in Model 1. At each time step, each agent's disease state is updated by application of the method Method_Update_Agent_Disease State to each agent. The logic within the update method is shown in Figure 2.

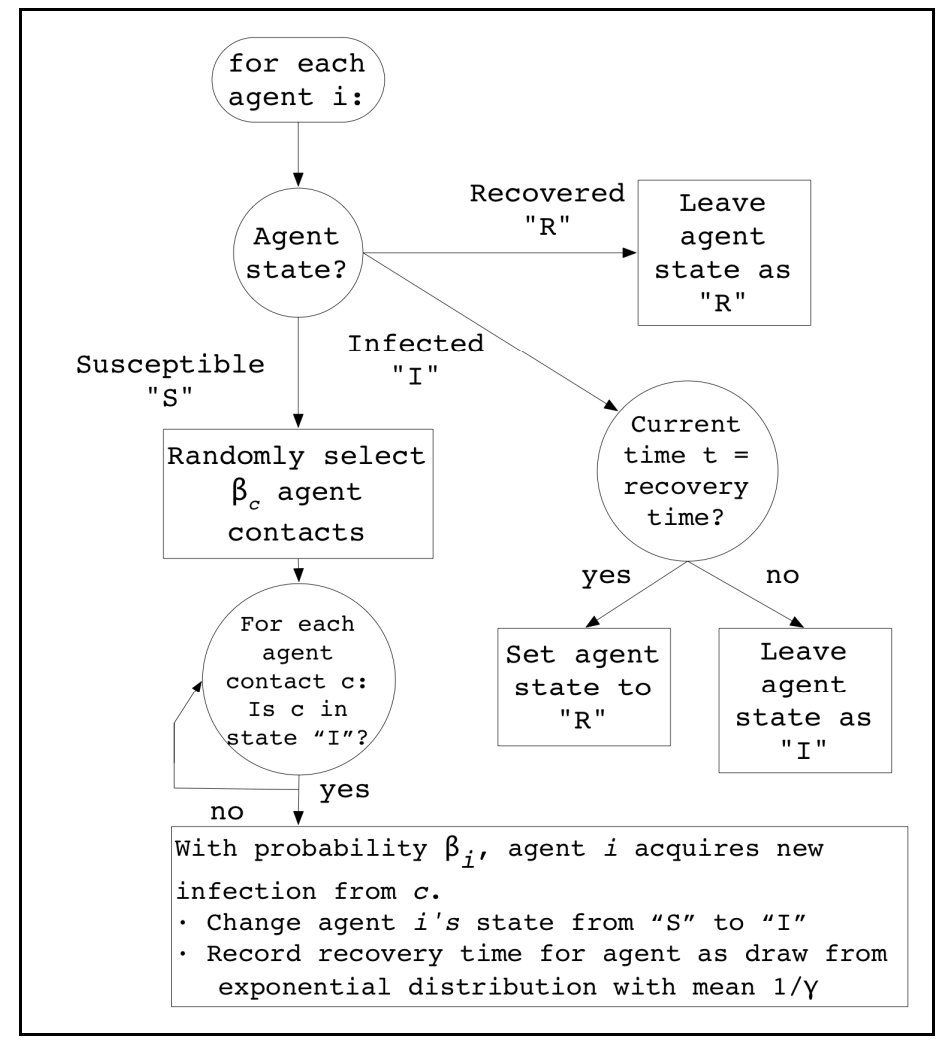

Figure 2: Logic of update method in AB Model 2 for updating the agent disease states at each time step 


\section{Macal}

\section{RESULTS}

\subsection{Model Comparison}

In this section we compare the results from the differential equation, System Dynamics and agent-based SIR models. Figure 3 shows the comparison of the results for the number of infected individuals over time. Note the results from the modeling approaches are similar but not exact. The mean of the ABS Model 2 runs deviates considerably from the median results from Model 2 as well as the results from the other models. This is due to the fact that Model 2 is able to explicitly capture the inherent stochasticity of the system that is embedded in SIR formulation, specifically in the parameters $\beta_{c}, \beta_{i}$, and $\gamma$ that are treated as random variables in ABMS Model 2.

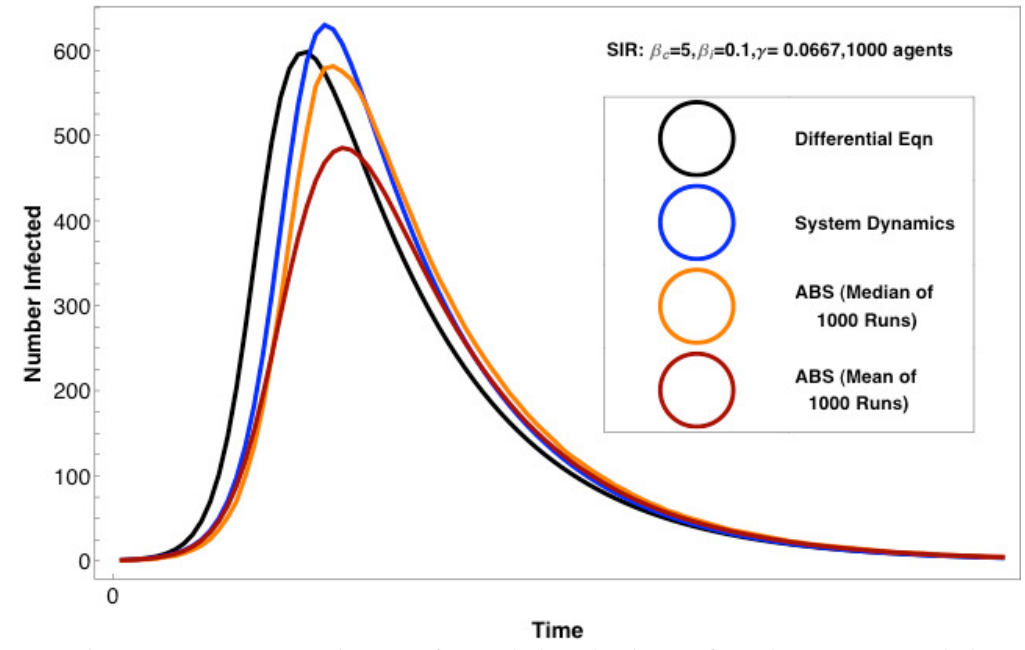

Figure 3: Comparison of model solutions for the SIR model

\subsection{Stochastic Variability}

Model 2 is stochastic in nature, in contrast to the differential equation, System Dynamics and agent-based Model 1, which are all deterministic. The range of stochastic variation in the results of the agent-based Model 2 for the number of infected individuals is shown in Figure 4, and compared to the deterministic solutions from the other modeling approaches. There is sizable variation across the Model 2 runs. A 70\% envelopes contains the results from both the differential equation and System Dynamics models.

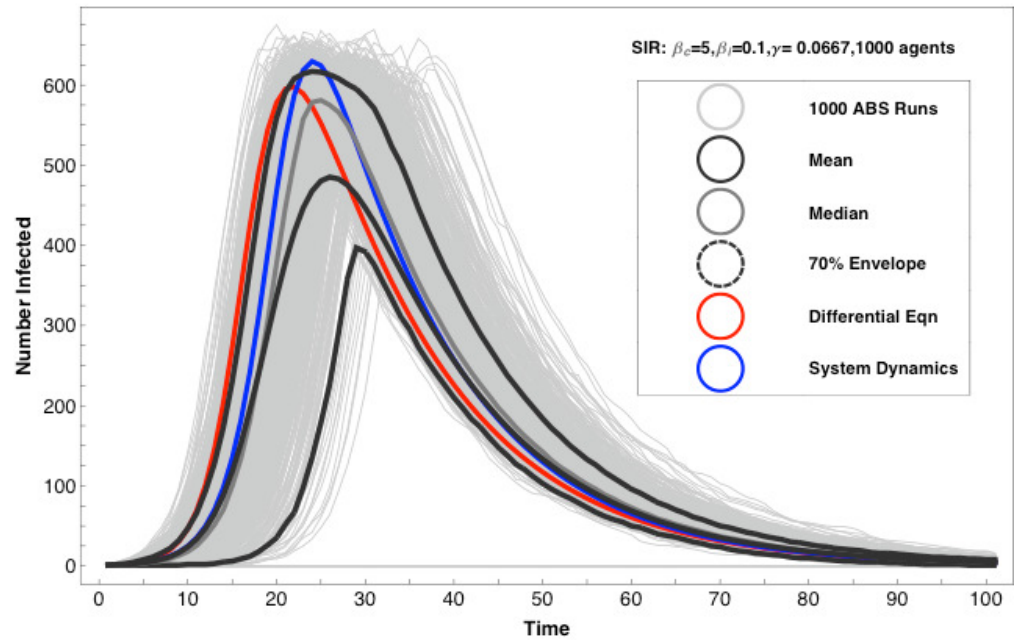

Figure 4: Variability in number of infected individuals across 1000 runs of the ABS SIR Model 2 


\section{Macal}

Figures 5 and 6 further elaborate and explain these results. Figure 5 shows the distribution of the peak numbers of infected individuals is bi-modal. This is because in some simulation runs of Model 2, an epidemic does not occur. In a small but significant number of cases, the number of contacts and the number of infecteds are not large enough to cause an increasing number of infected individuals, and the number of infections dies away before time 10 (Figure 6).

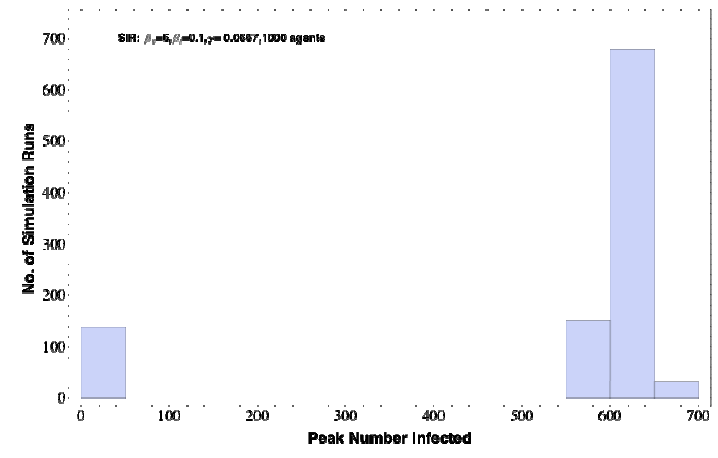

Figure 5: Distribution of Peak Infected Individuals

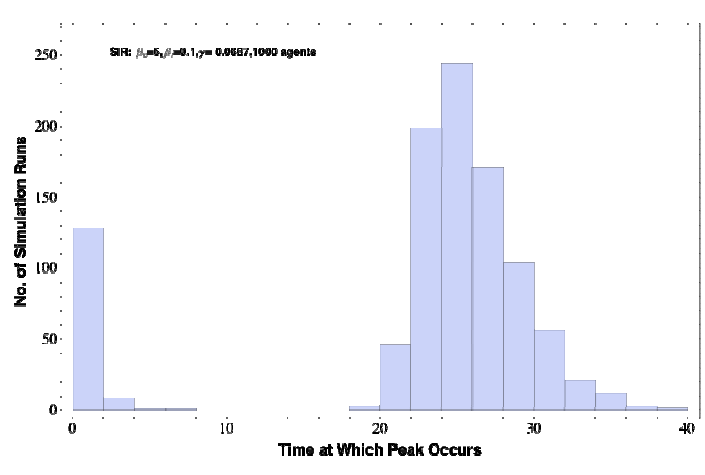

Figure 6: Distribution of Peak Infect Times

\section{CONCLUSION}

In this paper we have shown how to translate a System Dynamics model, which is deterministic, into an equivalent time-stepped, stochastic agent-based simulation. Specifically, probabilistic elements in the System Dynamics model are identified, isolated and translated into probabilities that are used explicitly in the agent-based model. For the SIR epidemic model considered here, the two probabilities were related to agent contact and to agent transmission of infection. The equivalency of the model results is not exact in terms of numerical accuracy for the reasons noted. We have shown that the agent-based model is able to provide additional information over what the System Dynamics model provides due to the explicit stochastic nature of the agent-based model.

\section{ACKNOWLEDGMENTS}

I would like to thank Andrew P. Wang for valuable research assistance and Vanja Dudic for discussions on epidemic modeling. This work was supported by the U.S. Department of Energy under contract number DE-AC02-06CH11357.

\section{REFERENCES}

Aleman, D. M., T. G. Wibisono, and B. Schwartz. 2009. Accounting for Individual Behaviors in a Pandemic Disease Spread Model. in Proceedings of the 2009 Winter Simulation Conference, ed. M. D. Rossetti, R. R. Hill, B. Johansson, A. Dunkin and R. G. Ingalls, 1977-1985. Piscataway, New Jersey: Institute of Electrical and Electronic Engineers, Inc.

Ascher, U. M., and L. R. Petzold. 1998. Computer methods for ordinary differential equations and differential-algebraic equations. SIAM: Society for Industrial and Applied Mathematics.

Bagni, R., R. Berchi and P. Cariello. 2002. A comparison of simulation models applied to epidemics, Journal of Artificial Societies and Social Simulation 5(3). http://jasss.soc.surrey.ac.uk/5/3/5.html.

Bonabeau, E. 2001. Agent-based modeling: methods and techniques for simulating human systems. In Proceedings of National Academy of Sciences 99(3): 7280-7287. 


\section{Macal}

Borshchev, A., and A. Filippov. 2004. From system dynamics and discrete event to practical agent based modeling: reasons, techniques, tools. The 22nd International Conference of the System Dynamics Society, July 25-29, 2004, Oxford, England.

Epstein, J. M. and R. Axtell, 1996, Growing artificial societies: social science from the bottom up, MIT Press: Cambridge, MA.

Epstein, J. M., J. Parker, et al. 2008. Coupled contagion dynamics of fear and disease: mathematical and computational explorations. PLoS ONE 3(12): e3955.

Forrester, J. W., 1961. Industrial dynamics, MIT Press: Cambridge, MA.

Forrester, J. W., 1971. Principles of systems, Wright-Allen Press (available from Pegasus Communications: Waltham, MA): Cambridge, MA.

Kermack, W. O. and A. G. McKendrick. 1927. Contributions to the mathematical theory of epidemics. Proc. Roy. Soc., To 15, (Part I, 1927).

Larson, R. 2007. Simple models of influenza progression within a heterogeneous population. Operations Research 55 (3): 399-412.

Letellier, C., S. Elaydi, L. A. Aguirre, and A. Alaoui. 2004. Difference equations versus differential equations, a possible equivalence for the Rössler system? Physica D. 195:29-49.

Macal, C., 2006. Methodological comparison of agent-based and system dynamics simulation, Proc. 2006 North American Association for Computational Social and Organizational Science (NAACSOS) Conference, South Bend, IN, June 22-24, available on CD.

Macal, C., and M. J. North. 2009. Agent-based modeling and simulation, in Proceedings of the 2009 Winter Simulation Conference, ed. M. D. Rossetti, R. R. Hill, B. Johansson, A. Dunkin and R. G. Ingalls, 86-98, Piscataway, New Jersey: Institute of Electrical and Electronic Engineers, Inc.

Marin, M., Y. Zhu, P. T. Meade, M. Sargent, and J. Warren. 2006. In Proceedings of the 2006 Winter Simulation Conference, eds. L. R. Perrone, F. P. Wieland, J. Liu, B. G. Lawson, D. M. Nicol, and R. M. Fujimoto, 667-671. Piscataway, New Jersey: Institute of Electrical and Electronics Engineers, Inc.

Mickens, R. E. 2002. Nonstandard finite difference schemes for differential equations, J. Diff. Eqns. Appl. 8(9):823-947.

Norling, E. 2007. Contrasting a system dynamics model and an agent-based model of food web evolution. in Multi-Agent-Based Simulation VII. pp. 57-68. Lecture Notes in Computer Science, Springer Berlin / Heidelberg. Volume 4442/2007.

North, M. J., and C. M. Macal. 2007. Managing business complexity: discovering strategic solutions with agent-based modeling and simulation, Oxford University Press: Oxford, U.K.

North, M. J., C. M. Macal. 2009. Agent-based modeling and systems dynamics model reproduction, International Journal of Simulation Process Modeling 5(3): 256-271.

Parunak, H. V. D., R. Savit, and R. L. Riolo, Agent-based modeling vs. equation-based modeling: a case study and users' guide. Proceedings of Workshop on Modeling Agent Based Systems (MABS98), Paris, 1998.

Rahmandad, H., and J. Sterman. 2008. Heterogeneity and network structure in the dynamics of diffusion: comparing agent-based and differential equation models. Management Science. 54(5): 998-1014.

Roberts, N., D. Anderson, R. Deal, M. Garet, and W. Schaffer. 1983. Introduction to computer simulation:a system dynamics modeling approach. Addision-Wesley.

Scholl, H. J. 2001. Agent based and system dynamics modeling: a call for cross study and joint research, 34th Annual Hawaii International Conference on System Sciences ( HICSS-34)-Volume 3, pp. 3003.

Sterman, J. D., 2000, Business Dynamics: Systems Thinking and Modeling for a Complex World, Boston: Irwin McGraw-Hill.

Wakeland, W. W., and E. J. Gallaher, L, M. Macovsky, and C. A. Aktipis. 2004. A comparison of system dynamics and agent-based simulation applied to the study of cellular receptor dynamics. 37th Annual Hawaii International Conference on System Sciences ( HICSS-37). 


\section{AUTHOR BIOGRAPHY}

CHARLES M. MACAL, Ph.D., P.E., is the Director of the Center for Complex Adaptive Agent Systems Simulation, Argonne National Laboratory, Senior Fellow in the joint Computation Institute of the University of Chicago and Argonne, and Adjunct Professor in the Graham School of the University of Chicago. $\mathrm{He}$ is a member of the INFORMS Simulation Society, the Association for Computing Machinery, the Society for Computer Simulation International, the Systems Dynamics Society and a founding member of the Computational Social Simulation Society. Dr. Macal has a Ph.D. in Industrial Engineering \& Management Sciences (Operations Research) from Northwestern University and a Master's Degree in Industrial Engineering (Computer Simulation) from Purdue. He is also a Registered Professional Engineer. His email address is $<$ macaleanl.gov $>$. 